\title{
Fermented foods and probiotics for children. The importance of knowing their microbiological differences
}

Dairy Science

(Instituto de Lactología

Industrial, INLAIN,

National Scientific

and Technical

Research Council

[Consejo Nacional

de Investigaciones

Científicas y

Técnicas, CONICET],

Universidad Nacional

del Litoral [UNL]),

Faculty of Chemical

Engineering,

Universidad Nacional del Litoral.

b. Department of Mother and Child Health of Hospital Militar Central de Buenos

Aires.

E-mail address:

Gabriel Vinderola, M.D.

gvinde@fiq.unl.edu.ar

\section{Funding:}

Project PICT-2016-0256

(National Agency of

Scientific and Technological

Promotion [Agencia Nacional

de Promoción Científica y

Tecnológica, ANPCyT]).

"Development of biological

cultures from native strains:

towards autonomy in the

technological production of

microorganisms of interest

in foods for humans and

animals," 2017-2020.

Conflict of interest:

$\mathrm{GV}$ is a principal investigator

at the National Scientific

and Technical Research

Council (Consejo Nacional

de Investigaciones Científicas

y Técnicas, CONICET) and

an Associated Professor

of the Microbiology Chair

at the Faculty of Chemical

Engineering (Facultad de

Ingeniería Química, FIQ) of

Universidad Nacional del

Litoral (UNL). GPM is the

principal investigator of several

clinical studies sponsored by

the pharmaceutical industry.

Both authors have performed

technological linkage tasks

(technological services, project

development, consultancy,

and counseling) with several

food industries. These

activities are considered

intellectually independent

from scientific manuscript

writing and had no effect on

the contents of this study.

Received: 5-20-2020

Accepted: 7-30-2020

\author{
Gabriel Vinderola, M.D. ${ }^{a}$ and Gonzalo Pérez-Marc, M.D. ${ }^{b}$
}

\begin{abstract}
Breast milk provides microorganisms that colonize the gut and program the immune system to develop oral tolerance. Between the 6 months of exclusive breastfeeding and the recommended 2 years of prolonged breastfeeding, complementary feeding leads to a progressive reduction in the entry of live microorganisms into the gut ecosystem. This is because foods in general-unlike breast milk-are devoid of microorganisms or, if present, they are often inactivated during cooking. Fermented foods and probiotics could be a valuable nutritional strategy, as they would ensure the supply of live microorganisms in the face of a reduction or early cessation of breastfeeding. The terms "fermented foods" and "probiotics" are not synonymous. Microbiological identity, safety, and the existence of clinical efficacy studies supporting both are key to understand their differences and decide on an eventual dietary recommendation.
\end{abstract}

Keywords: microbiota, immunity, yogurt, probiotics, fermented foods.

http: / / dx.doi.org/10.5546/ aap.2021.eng.56

To cite: Vinderola C, Pérez-Marc G. Fermented foods and probiotics for children. The importance of knowing their microbiological differences. Arch Argent Pediatr 2021;119(1):56-61.
Gut microbiota in the first $\mathbf{1 0 0 0}$ days

A child's first 1000 days of life is the period between fertilization and the age of 2 years. Throughout this period (and even before), and during our lifetime, human physiology is affected by microorganisms in different manners. It is even possible that the sperm responsible for our origin interacted with bacteria while traveling through the vaginal canal, and that fertility had been conditioned by the microbiota present there. ${ }^{1}$

The term "microbiota" refers to the set of microorganisms colonizing the body. The microbiota is mainly made up of bacteria, but it also includes a large amount of viruses, fungi, yeasts, protozoa, and archaea. The gut microbiota is the most studied community and the one with the greatest diversity and abundance of bacterial species. The term "microbiome" refers to the genes of the microorganisms found in the microbiota. Although the term "microbiota" encompasses "microbiome," only the former will be used in this article.

The microbiota is a "diffuse organ" inherited from the mother, the family setting, and the environment, which is established and becomes microbiologically mature during the first 2-3 years of life. In the context of a healthy and normal pregnancy, ${ }^{2}$ microbial material (non-viable cells, cell walls, and deoxyribonucleic acid [DNA]) has been reported to be present in the placenta, amniotic fluid, fetal membranes, and fetal gastrointestinal tract. However, the main maternal microbial contribution to the baby's gut colonization takes place with the passage through the vaginal canal and 
with breast milk. Other factors that complete the baby's gut colonization are the interaction with the family and the environment, as well as the type of feeding. ${ }^{3}$

Since breast milk is rich in nutrients, microorganisms, and oligosaccharides (formerly known as "bifidogenic factors"), it is the best possible nourishment for the development of gut microbiota and the gut immune maturation. ${ }^{4}$ The rapid establishment of a strictly anaerobic microbiota has important effects on the gut health due to the production of short-chain fatty acids; butyric acid is a major anti-inflammatory agent. ${ }^{5}$ Preterm birth, for example, causes a significant delay in the predominance of strict anaerobes. ${ }^{6}$ C-section birth and antibiotic use are associated with a higher prevalence, throughout childhood, of asthma, juvenile arthritis, inflammatory bowel disease, immune deficiency, overweight, obesity, allergies, eczema, and enteric and respiratory infections. $^{7}$

The mechanisms that would account for such inflammatory and immune disorders are related to the gut aberrant colonization, where (instead of the usual and beneficial Lactobacillus, Bifidobacterium or Faecalibacterium prausnitzii), proinflammatory microorganisms would prevail, including Enterococcus faecalis, Clostridium difficile, Campylobacter, and Methanobrevibacter smithii. ${ }^{3}$ Bifidobacterium is the prevalent genus in a healthy baby's gut during the first year of life. Its gut colonization dynamics is affected in children born via by $\mathrm{C}$-section since it becomes slower in the first months after birth. ${ }^{8}$ Such slowdown is critical in the development of the immune system, so a C-section birth would have an important impact on the predisposition to developing immune disorders. ${ }^{9}$ A recent publication in Archivos Argentinos de Pediatría highlights the importance of promoting breastfeeding, encouraging a rational antibiotic use in the perinatal period, and reducing the number of C-section births due to their clear effects on the newborn infant's microbiota. ${ }^{10}$

\section{The nutritional concept of a daily microorganism dose}

In 1907, immunologist and Nobel laureate Ilya Metchnikoff published a treatise on longevity, titled The Prolongation of Life: Optimistic Studies. Based on a series of observations, he proposed that Bulgarian peasants, who used to consume copious amounts of fermented dairy products, lived long and healthy lives. He attributed such longevity to the benefits of yogurt bacteria. ${ }^{11}$ This was the origin of the name
Lactobacillus bulgaricus, one of the two bacterial species found in yogurt.

A routine microorganism intake may provide multiple benefits, such as health maintenance and disease prevention. ${ }^{12}$ Breast milk, which contains up to $10^{7}$ microorganisms per milliliter, ${ }^{13}$ is the first microorganism-rich food to which we are exposed. Due to cultural issues, there is a strong belief that consuming a relatively small number of pathogenic microorganisms, sometimes present in food, could cause infections of varying severity, but, in a way, there is still skepticism about the contrary: if consumed in adequate amounts, certain microorganisms may be highly beneficial for health.

Our ancestors' nutrition was rich in microorganisms. However, the urbanization of our eating habits has significantly reduced our daily exposure to microbial-rich foods. ${ }^{14}$ Such reduced exposure to microorganisms has been associated with the development of chronic conditions. ${ }^{15}$ Therefore, how can we make a generation who learned about the importance of hygiene and cleanliness, for whom the only good microorganism is the one that is dead, understand the relevance of their presence in our usual diet? One way may be to adapt the concept of Recommended Dietary Allowance (RDA), which is the average intake of a specific nutrient a healthy person should consume on a daily basis to maintain an adequate health. In this setting, we may refer to a "microorganism RDA", just as that of macronutrients, vitamins, and trace elements (https: / / isappscience.org/rda-for-microbes/). Thus, we may wonder: What foods may offer safe RDAs for microorganisms as part of a child's complementary feeding?

\section{Fermented foods and probiotics}

The terms "probiotic" and "fermented food" have been used interchangeably, especially in social media. This is incorrect and may lead to doubts or confusion among health care professionals (nutritionists, gastroenterologists, pediatricians, geriatricians, etc.) when making dietary recommendations about these products. Not all fermented foods are probiotics and not all probiotics are exclusively available in fermented foods.

Fermented foods are those obtained through a controlled development of microorganisms (bacteria, yeasts, fungi) and their enzymatic action on food substrate, thus transforming it into a product different from the source material. 
Fermentation changes the rheological (viscosity) and sensory (smell, taste) properties of a product, improves the digestibility and bioavailability of some nutrients, extends its lifespan (due to the production of lactic acid), and may give certain health beneficial properties to food. ${ }^{16,17}$ Fermented foods may result from the fermentation of milk (yogurt, kefir), vegetables (sauerkraut, kimchi), cereals (beer, fermented oatmeal), and legumes or fruits (alcoholic beverages). The nutritional profile of fermented foods is highly diverse, and a comprehensive analysis is out of the scope of this study. From a microbiological perspective, fermentation may occur from microorganisms naturally present in the substrates to be fermented (sauerkraut, kimchi), through microbial consortia present in grains or "mothers" added to the substrate to be fermented (kefir, kombucha) or through selected microbial cultures (yogurt, cheese).

On their side, probiotics were defined by the World Health Organization in 2002 as "live microorganisms which, when administered in adequate amounts, confer a health benefit on the host". This definition was ratified by a group of experts of the International Scientific Association for Probiotics and Prebiotics (ISAPP) ${ }^{18}$ and introduced into the Argentine Food Code in 2011. The definition of "probiotic" involves three aspects: 1) the fact that it is a microorganism, or mix of microorganisms, with microbiological identity, i.e., having a known genus, species, and strain designation; 2 ) it is a live microorganism at the time of consumption; and 3) there is at least one clinical effectiveness study demonstrating its beneficial effects. ${ }^{19}$ Probiotics may be present in the form of dietary supplements (capsules, pills or aqueous suspensions) or introduced in foods, such as infant formulas, yogurts, fresh cheese or fruit juice. ${ }^{20}$

\section{Yogurt and yogurt with probiotics}

Yogurt is the product of milk fermentation with two specific bacteria, known as Streptococcus thermophilus and Lactobacillus delbrueckii, subsp. bulgaricus. They are responsible for partially fermenting lactose to acidify the milk. In general, yogurts are better tolerated than milk by subjects with lactose malabsorption or intolerance because part of the lactose is consumed during fermentation. In turn, an additional lactose hydrolysis takes place once the yogurt is consumed; lactose keeps on being hydrolyzed by the lactase enzyme released by lactic acid bacteria when lysed by biliary fluids.

Anyway, there are industrial lactose-free yogurts for people with high lactose intolerance. It is even possible to make homemade yogurts based on lactose-free milk because lactic acid bacteria are capable of finding, in glucose and galactose (sugars from lactose hydrolysis in industrial lactose-free milk), the necessary sugars to perform fermentation. It is worth noting that, thanks to the pasteurization processes that milk undergoes before fermentation, yogurt is not a potential vehicle for the bacteria that causes hemolytic uremic syndrome. ${ }^{21}$

In some cases, yogurt's microbiological composition may be enriched by adding probiotic bacteria, which are specific strains of the Lactobacillus and Bifidobacterium genera; L. casei, L. paracasei, L. rhamnosus or B. lactis are the most commonly used species. The microbial species used to make yogurt (or used as probiotics) have a Generally Recognized as Safe (GRAS) status according to the United States Food and Drug Administration (FDA) or a Qualified Presumption of Safety (QPS) status as per the European Food Safety Authority (EFSA).

An example of probiotic yogurt is that developed by the investigators of the Lactobacilli Reference Center (Centro de Referencia para Lactobacilos, CERELA), in Tucumán, and by different governmental institutions for use in a social program that started in the province of Tucumán and that has been implemented in schools across the Northwest region of Argentina (NOA) and several municipalities of Buenos Aires for more than a decade. ${ }^{22}$ This is an example of coincidence between the terms "fermented food" and "probiotic" because the product is the result of milk fermentation with Streptococcus thermophilus and Lactobacillus delbrueckii subsp. bulgaricus, followed by the addition of the probiotic Lactobacillus rhamnosus CRL1505, whose effectiveness has been, as applicable, studied in children for the prevention of respiratory tract infections, among other effects.

This is an example of those cases that adequately meet the definition of probiotic: there is a defined strain, it is possible to check its viability using specific culture media, ${ }^{23,24}$ and there is a clinical effectiveness study supporting its indication. ${ }^{25}$ Since it is included in a yogurt, this product may be referred to as a "probiotic fermented food." Another type of non-dairy probiotic fermented food is a product available in Nordic countries: fermented oatmeal containing 
the Lactobacillus plantarum $299 \mathrm{v}$ probiotic strain, ${ }^{26}$ which is then combined with different fruit pulps to make smoothies. ${ }^{27}$

A study demonstrated that introducing yogurt to diet provided more than 1000 times more microorganisms than a diet with no fermented foods. ${ }^{28}$ Probiotics may also be included in non-fermented foods, ${ }^{29}$ such as certain infant formulas ${ }^{30}$ or dietary supplements. ${ }^{31}$ In addition to Lactobacillus and Bifidobacterium, these also include other species, such as Lactobacillus reuteri, Saccharomyces boulardii or Bacillus coagulans. In relation to pediatric management, different probiotics have been developed that showed varying levels of effectiveness in the prevention of antibiotic-associated diarrhea ${ }^{32}$ and infantile colic. $^{33}$

International scientific societies have issued guidelines on the use of probiotics, like the World Gastroenterology Organization (https: / / www. worldgastroenterology.org/guidelines / globalguidelines / probiotics-and-prebiotics / probioticsand-prebiotics-english), the European Society for Pediatric Gastroenterology and Nutrition, and more recently, the American Gastroenterological Association. ${ }^{34}$

\section{Fermented foods with undefined microbiota}

Fermented foods, like milk kefir, water kefir, kombucha, sauerkraut or kimchi, are considered foods with an undefined consortium of microbes from a microbiological perspective because they are prepared from the spontaneous fermentation of the source material (with the bacteria and yeasts naturally present in the substrate) or, as in the case of kefir and kombucha, from a complex microbial community associated with kefir grains ${ }^{35}$ or the kombucha "mother" (SCOBY). ${ }^{17}$ These fermented foods contain a varying number of species and strains of lactic acid bacteria, acetic acid bacteria, and yeasts, as applicable. Kefir grains from different sources have a different microbiological composition, ${ }^{36}$ so they produce foods with a varying microbiological composition.

In addition, over the subsequent culture passages (or grain subcultures), the microbial community progressively changes its composition. ${ }^{37}$ In this context, it is not possible to know in advance the microorganisms present in homemade kefir. In addition, it is worth considering that both milk kefir and water kefir may contain $0.5-3 \%$ of alcohol due to the presence of yeasts, ${ }^{35,38,39}$ so their consumption is often discouraged during pregnancy, breastfeeding, and childhood. The identity of microorganisms in a product presented as "probiotic" is one of the requirements to know which microorganism it contains and, based on this, determine which studies support its probiotic capability. However, the fact that fermented foods with an indefinite composition (like kefir, sauerkraut or kombucha) cannot be technically called "probiotics" does not mean that they lack potential beneficial effects for health. At most, such effects may be inferred from studies done in similar foods, ${ }^{35}$ but this would not prevent the existence of doubts regarding the possibility of assigning them the same conclusions in terms of safety.

\section{CONCLUSION}

Breast milk is the best food for babies both due to its nutrient composition and the microorganisms it provides, which will make up the newborn infant's microbiota. Microorganisms play a key role on the modulation of the gut mucosal immune response for the development of oral tolerance and chronic disease prevention. Complementary feeding entails a reduction in the flow of live microorganisms to the gut, especially if breastfeeding is discontinued early. An excessive antibiotic use during early childhood and the increasing number of $\mathrm{C}$-section births have resulted in an alteration of microbiota abundance and diversity in infants. Because of this, the introduction of microorganisms in food may occupy, at least temporarily, the gut ecological niches and perform some natural functions of the microbiota indigenous members.

Both probiotics and fermented foods may be a source of safe and known microorganisms with great benefits at a local (gut) and systemic level. The terms "probiotic" and "fermented food" are not always interchangeable. Certain yogurts and infant formulas have probiotics. In these cases, it is possible to establish the microbiological identity of these products. Both yogurt microorganisms and cultures supported by clinical trials demonstrating their probiotic nature present in yogurt or infant formulas have a GRAS or QPS safety status. Microbiological identity and safety are important factors that health care professionals should take into account when making dietary recommendations for the introduction of live microorganisms to complementary feeding during early childhood. 


\section{REFERENCES}

1. García-VelascoJA, Menabrito M, Catalán IB. What fertility specialists should know about the vaginal microbiome: a review. Reprod Biomed Online. 2017; 35(1):103-12.

2. Kuperman AA, Koren O. Antibiotic use during pregnancy: How bad is it? BMC Med. 2016; 14(1):91.

3. Milani C, Duranti S, Bottacini F, Casey E, et al. The First Microbial Colonizers of the Human Gut: Composition, Activities, and Health Implications of the Infant Gut Microbiota. Microbiol Mol Biol Rev. 2017; 81(4):e00036-17.

4. Doare KL, Holder B, Bassett A, Pannaraj PS. Mother's Milk: A purposeful contribution to the development of the infant microbiota and immunity. Front Immunol.2018; 9:361.

5. Houghteling PD, Walker WA. Why is initial bacterial colonization of the intestine important to infants' and children's health? J Pediatr Gastroenterol Nutr. 2015; 60(3):394-307.

6. Arboleya S, Solís G, Fernández N, De los Reyes-Gavilán $\mathrm{CG}$, et al. Facultative to strict anaerobes ratio in the preterm infant microbiota: A target for intervention? Gut Microbes. 2012; 3(6):583-8.

7. Tamburini S, Shen $\mathrm{N}, \mathrm{Wu} \mathrm{HC}$, Clemente JC. The microbiome in early life: Implications for health outcomes. Nat Med. 2016; 22(7):713-22.

8. Reyman M, Van Houten MA, Van Baarle D, Bosch AA, et al. Impact of delivery mode-associated gut microbiota dynamics on health in the first year of life. Nat Commun. 2019; 10(1):4997.

9. Francino MP. Birth Mode-Related Differences in Gut Microbiota Colonization and Immune System Development. Ann Nutr Metab. 2018; 73(Suppl 3):12-6.

10. Uberos J. Microbiota perinatal: Revisión de su importancia en la salud del recién nacido. Arch Argent Pediatr. 2020; 118(3):e265-70.

11. Reid G. Probiotics: Definition, scope and mechanisms of action. Best Pract Res Clin Gastroenterol. 2016; 30(1):17-25.

12. Sanders ME, Benson A, Lebeer S, Merenstein DJ, et al. Shared mechanisms among probiotic taxa: implications for general probiotic claims. Curr Opin Biotechnol. 2018; 49:207-16

13. Boix-Amorós A, Collado MC, Mira A. Relationship between milk microbiota, bacterial load, macronutrients, and human cells during lactation. Front Microbiol. 2016; 7:492.

14. Logan AC, Katzman MA, Balanzá-Martínez V. Natural environments, ancestral diets, and microbial ecology: Is there a modern "paleo-deficit disorder"? Part II. J Physiol Anthropol. 2015; 34(1):9.

15. Kramer A, Bekeschus S, Bröker BM, Schleibinger H, et al. Maintaining health by balancing microbial exposure and prevention of infection: The hygiene hypothesis versus the hypothesis of early immune challenge. J Hosp Infect. 2013; 83(Suppl 1):S29-34.

16. Di Cagno R, Coda R, De Angelis M, Gobbetti M. Exploitation of vegetables and fruits through lactic acid fermentation. Food Microbiol. 2013; 33(1):1-10.

17. Dimidi E, Cox SR, Rossi M, Whelan K. Fermented foods: Definitions and characteristics, impact on the gut microbiota and effects on gastrointestinal health and disease. Nutrients. 2019; 11(8):1806.

18. Hill C, Guarner F, Reid G, Gibson GR, et al. Expert consensus document: The international scientific association for probiotics and prebiotics consensus statement on the scope and appropriate use of the term probiotic. Nat Rev Gastroenterol Hepatol. 2014; 11(8):50614.

19. Reid G, Gadir AA, Dhir R. Probiotics: Reiterating what they are and what they are not. Front Microbiol. 2019; $10: 424$.

20. Fenster K, Freeburg B, Hollard C, Wong C, et al. The production and delivery of probiotics: A review of a practical approach. Microorganisms. 2019; 7(3):83.

21. Vinderola G, Rivas M. Síndrome Urémico Hemolítico y yogur: entre la creencia popular y la evidencia científica. Rev Chil Nutr. 2020; 47(1):148-52.

22. Bortza G, Thomas H. Biotechnologies for inclusive development: Scaling up, knowledge intensity and empowerment (the case of the probiotic yoghurt 'yogurito' in Argentina). Innov Dev. 2017; 7(1):37-61.

23. Vinderola CG, Reinheimer JA. Culture media for the enumeration of Bifidobacterium bifidum and Lactobacillus acidophilus in the presence of yoghurt bacteria. Int Dairy J. 1999; 9(8):497-505.

24. Vinderola G, Reinheimer J, Salminen S. The enumeration of probiotic issues: From unavailable standardised culture media to a recommended procedure? Int Dairy J. 2019; 96:58-95.

25. Villena JC, Salva MS, Nuñez MS, Corzo J, et al. Probiotics for Everyone! The Novel Immunobiotic Lactobacillus rhamnosus CRL1505 and the Beginning of Social Probiotic Programs in Argentina. Int J Biotechnol Wellness Ind. 2012; 1(3):189-98.

26. Molin G. Probiotics in foods not containing milk or milk constituents, with special reference to Lactobacillus plantarum 299v. Am J Clin Nutr. 2001; 73(2 Suppl):S380-5.

27. McNaught CE, Woodcock NP, Anderson ADG, MacFie J. A prospective randomised trial of probiotics in critically ill patients. Clin Nutr. 2005; 24(2):211-9.

28. Lang JM, Eisen JA, Zivkovic AM. The microbes we eat: Abundance and taxonomy of microbes consumed in a day's worth of meals for three diet types. Peer J. 2014; 2:e6590.

29. Hojsak I, Fabiano V, Pop TL, Goulet O, et al. Guidance on the use of probiotics in clinical practice in children with selected clinical conditions and in specific vulnerable groups. Acta Paediatr. 2018; 107(6):927-37.

30. Green Corkins K, Shurley T. What's in the Bottle? A Review of Infant Formulas. Nutr Clin Pract. 2016; 31(6):723-9.

31. Sreeja V, Prajapati JB. Probiotic Formulations: Application and Status as Pharmaceuticals-A Review. Probiotics Antimicrob Proteins. 2013; 5(2):81-91.

32. Szajewska H, Canani RB, Guarino A, Hojsak I, et al. Probiotics for the prevention of antibiotic-associated diarrhea in children. I Pediatr Gastroenterol Nutr. 2016; 62(3):495-506.

33. Urbańska M, Szajewska H. The efficacy of Lactobacillus reuteri DSM 17938 in infants and children: a review of the current evidence. Eur J Pediatr. 2014; 173(10):1327-37.

34. Su GL, Ko CW, Bercik P, Falck-Ytter Y, et al. AGA Clinical Practice Guidelines on the Role of Probiotics in the Management of Gastrointestinal Disorders. Gastroenterology. 2020; S0016-5085(20)34729-6.

35. Rosa DD, Dias MMS, GrześkowiakŁM, Reis SA, et al. Milk kefir: Nutritional, microbiological and health benefits. Nutr Res Rev. 2017; 30(1):82-96.

36. Plessas S, Nouska C, Mantzourani I, Kourkoutas Y, et al. Microbiological exploration of different types of Kefir grains. Fermentation. 2017; 3(1).

37. Gao W, Zhang L, Feng Z, Liu H, et al. Microbial 
diversity and stability during primary cultivation and subcultivation processes of Tibetan kefir. Int J Food Sci Technol. 2015; 50(6):1468-76.

38. Laureys D, De Vuyst L. Microbial species diversity, community dynamics, and metabolite kinetics of water kefir fermentation. Appl Environ Microbiol. 2014; 80(8):2564-72.

39. Laureys D, De Vuyst L. The water kefir grain inoculum determines the characteristics of the resulting water kefir fermentation process. J Appl Microbiol.2017; 122(3):719-732. 